\title{
Diseño y Experimentación de Actividades para el Aprendizaje en la Asignatura Psicología de Memoria del Grado de Psicología
}

\author{
Activities Design and \\ Experimentation for Learning \\ in the subject about Psychology \\ of Memory belonging to \\ Degree in Psychology
}

ESPERANZA QUINTERO SÁNCHEZ

ORCID: https://orcid.org/0000-0002-6878-9031

Universidad de Sevilla. Facultad de Psicología.

Departamento de Psicología Experimental

equintero@us.es

Fecha de recepción:

Fecha de aceptación:

DOI: http://dx.doi.org/10.12795/9788447221912.037

Pp.: 844-862 


\section{Resumen}

El Ciclo de Mejora (CIMA) desarrollado, ha sido una continuación y adaptación de un primer ciclo de iniciación, en la asignatura de la Psicología de la Memoria en el Grado de Psicología. Se ha pretendido la implantación de cursos y características de la Innovación Docente que se está desarrollando en ámbito de la docencia universitaria. Se ha pretendido y trabajado en torno a los contenidos de la asignatura no solo de tipo conceptual, sino también procedimentales y actitudinales, para la formación de futuros profesionales. Esto se ha llevado a cabo a través del trabajo de varias actividades (problemas de tipo práctico y experimental). Tras el análisis de los resultados obtenidos mediante la evaluación inicial y final del CIMA, puede comprobarse que esta nueva forma de practicar la docencia ha sido positiva.

Palabras Clave: CIMA, Innovación Docente, Contenidos conceptual, procedimental, actitudinal, Psicología de la Memoria, Grado de Psicología.

\section{Abstract}

The Cycle of Improvement (CIMA) developed, it has been a continuation and adaptation of a first cycle of initiation, in the subject of the Psychology of Memory in the Degree in Psychology. It has been tried the implantation of courses and characteristics of the Innovation Teaching that is being developed in the field of the university teaching. It has been tried and worked around the contents of the subject not only conceptual, but also procedural and attitudinal, for the training of future professionals. This has been carried out through the work of several activities (practical and experimental problems). After the analysis of the results obtained through the initial and final evaluation of the CIMA, it can be verified that this new way of teaching has been positive.

Keywords: CIMA, Teaching Innovation, Conceptual, procedural, attitudinal contents, Psychology of the Memory, Degree in Psychology 


\section{Introducción}

El Ciclo de Mejora llevado a cabo en el grado de Psicología, es una continuación de la formación empezada en 2017, dentro de los Ciclos de Mejora de la REFID, que ofrece el Instituto de Ciencias de la Educación (ICE), y que son ofrecidos a los docentes de la Universidad de Sevilla. Este ciclo ha permitido que el desarrollo de las clases sea más activo, lo que supone, al menos en parte, una pequeña transformación de la tradicional forma de impartir la docencia. En base, a lo obtenido en el anterior ciclo, en el que se implantó en una asignatura de Máster, en la que parte implicaba el estudio de esta asignatura; se propuso desarrollar el siguiente ciclo en una mayor extensión de la temática, si bien en este caso, en el grado de Psicología al que pertenece.

Asignatura en la que se va a aplicó fue: "Psicología de la Memoria" del Grado de Psicología, siendo una asignatura obligatoria del primer año; de modo que los estudiantes no cuentan con ninguna formación en la temática. La misma consta de 8,6 créditos por grupo, en el que he impartido docencia en un grupo completo. Esta asignatura tiene grupo grande, grupo medianos de dos horas y grupo pequeños de una hora. Así fue, en grupos medianos y pequeños donde implanté el Ciclo de Mejora; con un total de 7 días dónde se repartieron las actividades; y que se llevaron a cabo en aulas de la facultad cuyo mobiliario permitía el trabajo en grupo. La evaluación de estas áreas se hará mediante caso práctico incluido en el examen final, con un peso del $10 \%$ en el total de la asignatura; y con el desarrollo de un informe experimental de la reproducción de uno de los experimentos que trabajemos en clase, y que tendrá un peso del 30\% del total de la nota.

El objetivo de implantar este Ciclo de Mejora, fue el hecho de que trabaja con cuestiones prácticas o procedimentales, permite abarcar de una forma más amplia las 
temáticas a tratar y que de esta manera junto con los mapas de contenido, es posible trabajar los aspectos teóricos, procedimentales y actitudinales.

\section{Diseño previo del CIMA}

\section{Mapa conceptual}

Comenzando con el Mapa de Contenidos en el que nos basamos, este es una reformulación del trabajado en el ciclo anterior, y en que hay una relación directa entre las actividades que se trabajaron y los aspectos a trabajar.

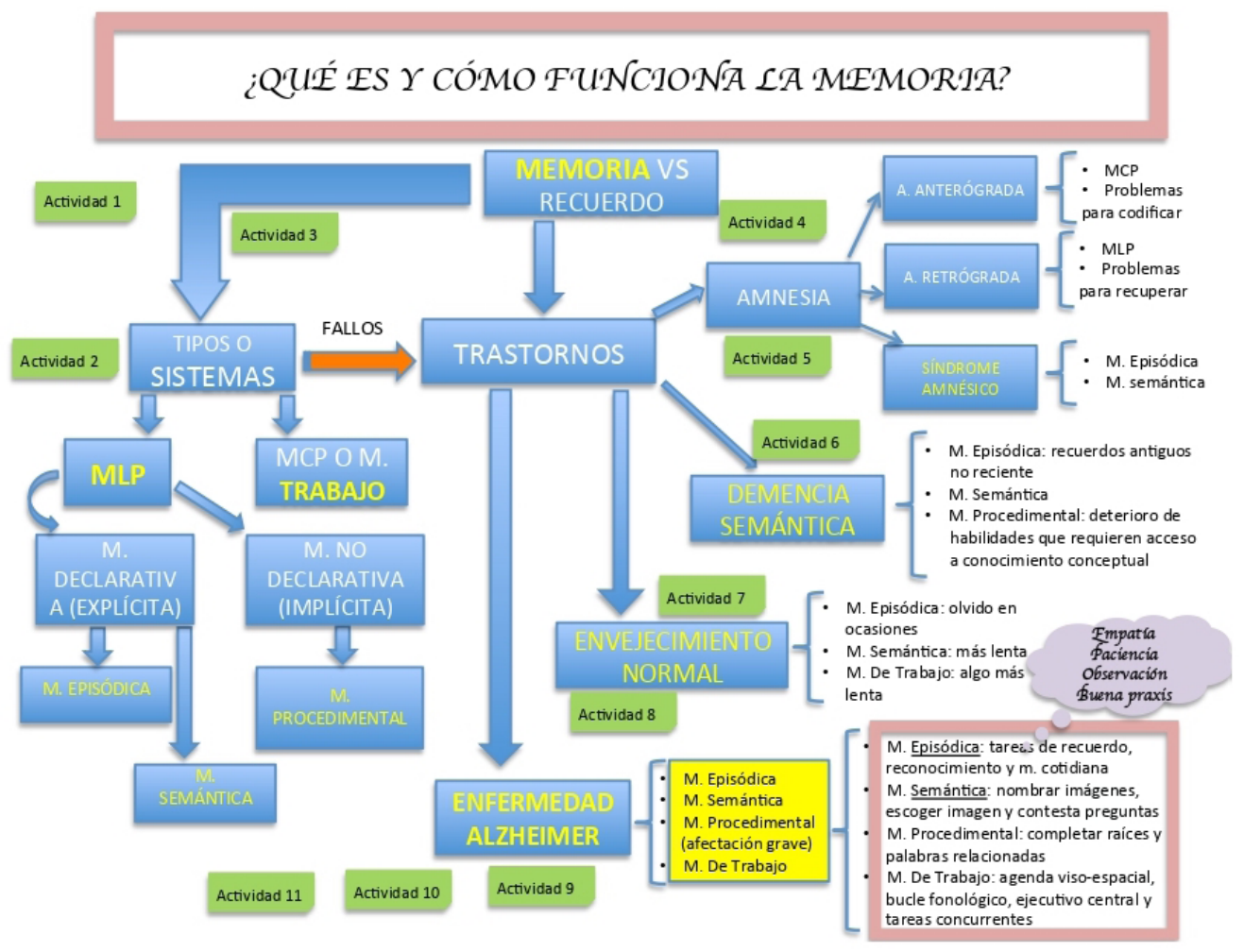

Figura 1. Mapa conceptual

Jornadas de Formación e Innovación Docente del Profesorado | № 2 (2019) Esta obra se distribuye con la licencia Creative Commons Reconocimiento-NoComercial-SinObraDerivada 


\section{Explicación mapa}

El mapa comienza planteando una pregunta, un problema, lo que atañe cuestiones tanto de tipos conceptual, como procedimental y actitudinal

- Lo que está en cuadros azules implica lo conceptual, y dentro de estos los conceptos amarillos son los

importantes a tener en cuanta para el problema planteado

- Con un recuadro rosa se destaca lo que implica procedimental

- Y con el globo morado y letras apple chancery las cuestiones actitudinales

- Las actividades que iremos realizando y con las que trabajaremos los contenidos conceptuales,

procedimentales y actitudinales, quedan recogidas en los cuadros verdes.

\section{Modelo metodológico y Secuencia de Actividades}

En cuanto al Modelo Metodológico seguido ha sido un modelo Práctico-Teórico, ya que partía de cuestiones prácticas para a raíz de ésta ir planteando y explicando los conceptos teóricos, pero también desgranar la parte procedimental y actitudinal que implica la Psicología de la Memoria, y que debe desarrollar un buen Psicólogo que trabaje en estas cuestiones. Es por ello, que las actividades han estado bien planteadas para desarrollar todos estos planteamientos.

Concretamente, me he basado en el Modelo de reelaboración de las ideas de los estudiantes, de manera que se pusieran en interacción los significados a los tres niveles de conocimiento con los que trabajamos y de los cuales partían; reelaborando los esquemas iniciales "activando sus mentes". Para ello realizamos actividades tanto de casos prácticos como experimentales; para ir desarrollando los diferentes conceptos teóricos (tanto conceptuales, como procedimentales y actitudinales).

El modelo seguiría un patrón:

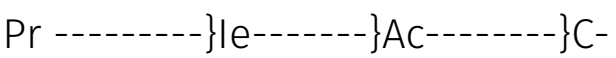

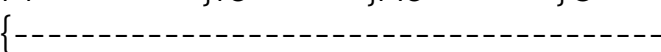

Pr: planteamiento del problema-caso-experimento 
le: toma de conciencia y expresión de los estudiantes de sus ideas del problema

Ac: puesta en práctica de las actividades de contraste (en común)

C: elaboración de conclusiones iniciales y finales del proceso de aprendizaje.

Las Actividades y la programación desarrollada se muestran en el siguiente cuadro:

Tabla 1. Secuencia de Actividades desarrolladas en Grupos Medianos

\begin{tabular}{|c|c|c|c|}
\hline ACTIVIDAD GM & DESCRIPCIÓN & CONTENIDO & DESARROLLO \\
\hline$\frac{1 \text { a ACTIVIDAD: }}{\text { EXPERIMENTO } 1}$ & $\begin{array}{l}\text { Realización del } \\
\text { experimento } \\
\text { “Disociación de } \\
\text { Memoria”, con las } \\
\text { distintas fases } \\
\text { que implica: } \\
\text { procesamiento } \\
\text { de la información } \\
\text { y medida de } \\
\text { recuperación. } \\
\text { Exposición de } \\
\text { datos obtenidos y } \\
\text { cómo trabajar con } \\
\text { ellos; así como } \\
\text { la interpretación } \\
\text { teórica de } \\
\text { los mismo; y } \\
\text { como realizar } \\
\text { un informe } \\
\text { experimental. } \\
\text { Tiempo: 120 } \\
\text { minutos }\end{array}$ & $\begin{array}{l}\text { Conceptos } \\
\text { teóricos, de los } \\
\text { diferentes tipos } \\
\text { de memoria } \\
\text { a largo plazo, } \\
\text { así como de } \\
\text { las medidas de } \\
\text { recuperación de } \\
\text { la información; } \\
\text { recogida de } \\
\text { datos, análisis y } \\
\text { desarrollo de las } \\
\text { diferentes partes } \\
\text { de un informe } \\
\text { experimental. }\end{array}$ & $\begin{array}{l}\text { Se ha llevado a } \\
\text { cabo sin problemas, } \\
\text { aunque al principio } \\
\text { se ha generado } \\
\text { algo de inquietud } \\
\text { en el alumnado, al } \\
\text { ser una actividad } \\
\text { nueva. }\end{array}$ \\
\hline
\end{tabular}

Jornadas de Formación e Innovación Docente del Profesorado | № 2 (2019) Esta obra se distribuye con la licencia Creative Commons Reconocimiento-NoComercial-SinObraDerivada Internacional (CC BY-NC-ND 4.0.) 


\begin{tabular}{|c|c|c|c|}
\hline $\begin{array}{l}\text { 2a ACTIVIDAD: } \\
\text { EXPERIMENTO } 2\end{array}$ & $\begin{array}{l}\text { Realización del } \\
\text { experimento } \\
\text { "Memoria y } \\
\text { Emoción”, con las } \\
\text { distintas fases } \\
\text { que implica: } \\
\text { medida de } \\
\text { estado emocional } \\
\text { línea base, } \\
\text { procesamiento de } \\
\text { la información, } \\
\text { inducción de la } \\
\text { emoción, medida } \\
\text { estado emocional } \\
\text { postinducción } \\
\text { y medida de } \\
\text { recuperación de } \\
\text { la información. } \\
\text { Exposición de } \\
\text { datos obtenidos y } \\
\text { cómo trabajar con } \\
\text { ellos; así como } \\
\text { la interpretación } \\
\text { teórica de } \\
\text { los mismo; y } \\
\text { como realizar } \\
\text { un informe } \\
\text { experimental. } \\
\text { Tiempo: 120 } \\
\text { minutos }\end{array}$ & $\begin{array}{l}\text { Conceptos } \\
\text { teóricos, de } \\
\text { la relación } \\
\text { entre memoria, } \\
\text { procesamiento y } \\
\text { recuperación de } \\
\text { la información } \\
\text { con las } \\
\text { emociones; } \\
\text { recogida de } \\
\text { datos, análisis y } \\
\text { desarrollo de las } \\
\text { diferentes partes } \\
\text { de un informe } \\
\text { experimental. }\end{array}$ & $\begin{array}{l}\text { Se ha llevado a } \\
\text { cabo sin problemas, } \\
\text { además en } \\
\text { esta ocasión el } \\
\text { alumnado se ha } \\
\text { mostrado más } \\
\text { colaborador }\end{array}$ \\
\hline
\end{tabular}

Jornadas de Formación e Innovación Docente del Profesorado | № 2 (2019) 


\begin{tabular}{|c|c|c|c|}
\hline $\begin{array}{l}\text { 3a ACTIVIDAD: } \\
\text { EXPERIMENTO } 3\end{array}$ & $\begin{array}{l}\text { Realización del } \\
\text { experimento } \\
\text { "Memoria y } \\
\text { Perspectiva } \\
\text { cognitiva", con las } \\
\text { distintas fases } \\
\text { que implica: } \\
\text { procesamiento } \\
\text { de la información } \\
\text { desde una } \\
\text { perspectiva } \\
\text { cognitiva } \\
\text { concreta, tarea } \\
\text { distractora, } \\
\text { recuperación de } \\
\text { la información } \\
\text { desde la } \\
\text { perspectiva } \\
\text { cognitiva } \\
\text { contraria. } \\
\text { Exposición de } \\
\text { datos obtenidos y } \\
\text { cómo trabajar con } \\
\text { ellos; así como } \\
\text { la interpretación } \\
\text { teórica de } \\
\text { los mismo; y } \\
\text { como realizar } \\
\text { un informe } \\
\text { experimental. } \\
\text { Tiempo: 120 } \\
\text { minutos }\end{array}$ & $\begin{array}{l}\text { Conceptos } \\
\text { teóricos, de la } \\
\text { relación entre } \\
\text { memoria y } \\
\text { perspectiva } \\
\text { cognitiva y su } \\
\text { influencia en la } \\
\text { recuperación de } \\
\text { la información; } \\
\text { recogida de } \\
\text { datos, análisis y } \\
\text { desarrollo de las } \\
\text { diferentes partes } \\
\text { de un informe } \\
\text { experimental. }\end{array}$ & $\begin{array}{l}\text { Se ha desarrollado } \\
\text { sin incidentes }\end{array}$ \\
\hline
\end{tabular}

Jornadas de Formación e Innovación Docente del Profesorado I № 2 (2019) 


\begin{tabular}{|c|c|c|c|}
\hline $\begin{array}{l}\frac{4 \underline{a}, 6 \underline{a} \text { Y } 7 \underline{a}}{\text { ACTIVIDAD: }} \\
\frac{\text { TRABAJO EN }}{\text { EL INFORME }} \\
\text { EXPERIMENTAL }\end{array}$ & $\begin{array}{l}\text { Realización } \\
\text { del informe } \\
\text { experimental } \\
\text { con las distintas } \\
\text { partes: título, } \\
\text { autoría, resumen; } \\
\text { introducción } \\
\text { teórica, teniendo } \\
\text { que haber hecho } \\
\text { búsqueda de } \\
\text { referencias } \\
\text { bibliográficas; } \\
\text { metodología, } \\
\text { explicitando el } \\
\text { desarrollo del } \\
\text { experimento que } \\
\text { hayan elegido de } \\
\text { los tres anteriores } \\
\text { realizados en } \\
\text { clase, y que han } \\
\text { tenido que hacer } \\
\text { de forma externa } \\
\text { a la facultad a } 30 \\
\text { sujetos; así como } \\
\text { el análisis de } \\
\text { datos; resultados; } \\
\text { conclusiones; } \\
\text { y referencias } \\
\text { bibliográficas } \\
\text { siguiendo normas } \\
\text { APA. Tiempo: } 120 \\
\text { minutos }\end{array}$ & $\begin{array}{l}\text { Conceptos } \\
\text { teóricos y } \\
\text { prácticos de } \\
\text { los aspectos } \\
\text { experimentales } \\
\text { y de la } \\
\text { investigación, } \\
\text { así como de } \\
\text { los diferentes } \\
\text { conceptos } \\
\text { teóricos; y todo } \\
\text { lo que implica } \\
\text { el desarrollo de } \\
\text { un informe de } \\
\text { investigación. }\end{array}$ & $\begin{array}{l}\text { Estás clases } \\
\text { has sido bien } \\
\text { aprovechadas por } \\
\text { los alumnos, ya } \\
\text { que si bien no son } \\
\text { obligatorias, se } \\
\text { las han planteado } \\
\text { como tutorías en } \\
\text { las que podían } \\
\text { aclarar dudas, } \\
\text { tanto con el } \\
\text { profesor como con } \\
\text { los compañeros, } \\
\text { y aprender más } \\
\text { sobre las tareas } \\
\text { que implican la } \\
\text { investigación en } \\
\text { psicología }\end{array}$ \\
\hline$\frac{\text { 5a ACTIVIDAD: }}{\frac{\text { ANÁLISIS DE }}{\text { DATOS }}}$ & $\begin{array}{l}\text { Análisis de } \\
\text { los datos } \\
\text { previamente } \\
\text { recogidos } \\
\text { mediante } \\
\text { programa SPSS. } \\
\text { Tiempo: } 120 \\
\text { minutos }\end{array}$ & $\begin{array}{l}\text { Conceptos } \\
\text { teóricos y } \\
\text { prácticos de } \\
\text { los aspectos } \\
\text { experimentales } \\
\text { y de la } \\
\text { investigación, en } \\
\text { concreto la parte } \\
\text { metodológica } \\
\text { de análisis } \\
\text { estadísticos. }\end{array}$ & $\begin{array}{l}\text { Esta ha sido } \\
\text { fundamentalmente } \\
\text { desarrollada por mí, } \\
\text { y posteriormente } \\
\text { explicada a ellos. } \\
\text { Si bien sería } \\
\text { interesante que } \\
\text { en futuros Ciclos } \\
\text { fuesen ellos los } \\
\text { que adquiriesen } \\
\text { también estos } \\
\text { contenidos. }\end{array}$ \\
\hline
\end{tabular}

Jornadas de Formación e Innovación Docente del Profesorado I № 2 (2019) 
Tabla 2. Secuencia de Actividades desarrolladas en Grupos Pequeños.

\begin{tabular}{|c|c|c|c|}
\hline ACTIVIDADES GP & DESCRIPCIÓN & CONTENIDO & DESARROLLO \\
\hline $\begin{array}{l}\frac{1 \underline{\mathrm{a}}, 2 \underline{\mathrm{a}}, 3 \underline{\mathrm{a}}, 4 \underline{\mathrm{a}},}{\frac{5 \underline{\mathrm{a}}, 6 \underline{\mathrm{a}} \text { y } 7 \underline{\mathrm{a}}}{\text { ACTIVIDAD: }}} \\
\frac{\text { ANÁLISIS DE }}{\text { CASO PRÁCTICOS }}\end{array}$ & $\begin{array}{l}\text { Análisis de casos } \\
\text { prácticos, a través } \\
\text { de visualización } \\
\text { de casos o en } \\
\text { textos con casos } \\
\text { descritos. Tiempo: } \\
60 \text { minutos (por } 7 \\
\text { días) }\end{array}$ & $\begin{array}{l}\text { Conceptos teóricos } \\
\text { y prácticos de los } \\
\text { distintos tipos } \\
\text { de memoria, } \\
\text { trastornos, así } \\
\text { como los métodos } \\
\text { de evaluación y } \\
\text { su aplicación, y } \\
\text { cómo interpretar } \\
\text { los resultados } \\
\text { y posterior } \\
\text { diagnosis. }\end{array}$ & $\begin{array}{l}\text { Se han ido } \\
\text { desarrollando } \\
\text { en complejidad, } \\
\text { desde una visión } \\
\text { más holística a } \\
\text { una más concreta, } \\
\text { trabajando en } \\
\text { común todos en el } \\
\text { aula, los diferentes } \\
\text { contenidos a } \\
\text { tres niveles } \\
\text { que implica el } \\
\text { trabajos con } \\
\text { pacientes. Si bien } \\
\text { sería interesante } \\
\text { trabajar más con } \\
\text { formato examen } \\
\text { de la asignatura. }\end{array}$ \\
\hline
\end{tabular}

\section{Cuestionario inicial-final para la evaluación pre y post ciclo de mejora en los alumnos}

Se planteó el mismo cuestionario, para que de esta manera pudiésemos analizar la evolución de las respuestas de los alumnos, así como el cambio de concepciones de los temas tratados; y que esto pudiese verlo reflejado en la escalera de conocimiento.

Nombre:

Responde las siguientes preguntas:

¿Qué es para ti la memoria? ¿Hay diferentes tipos?

¿Qué trastornos de memoria conoces? Enuméralos así como intenta describir sus características

Jornadas de Formación e Innovación Docente del Profesorado | № 2 (2019) Esta obra se distribuye con la licencia Creative Commons Reconocimiento-NoComercial-SinObraDerivada Internacional (CC BY-NC-ND 4.0.) 
¿Por qué crees que hay pérdida de memoria en las personas mayores? ¿Y en personas de mediana edad? ¿Y en personas que sufren algún tipo de "trauma" (psicológico, vascular, accidental, alcholismo...)?

¿Cómo sabrías si una persona tiene Alzheimer?

¿Cómo evaluarías e intervendrías a una persona con Alzheimer?

(NOTA: se escoge el trastorno de Alzheimer, por ser el más representativo de pérdida de todos los sistemas de memoria)

\section{Aplicación del CIMA}

\section{Relato de las sesiones y Escalera de Aprendizaje}

Lo sucedido durante el desarrollo de las sesiones, puede verse en los cuadros de las actividades planteadas del Ciclo, en el que se incluye una columna sobre el desarrollo, además de verse resumido en la Escalera de Aprendizaje (Figura 2 Y Tabla 3). No obstante paso a detallar de manera general tanto los avances como las dificultades encontradas.

Como suele ocurrir cuando se cambian las estructuras tradicionales de enseñanza, los alumnos tienden a mostrar resistencia, contamos además con la cuestión de ser alumnos de primer curso, donde todavía muestran más miedo ante los cambios y a los modelos aprendidos que traen. Lo cierto es que aplicar este nuevo tipo de enseñanza a través de los problemas, posteriormente, les resulta mucho más motivador, y la adquisición del contenido tanto conceptual, como procedimental y actitudinal, va siendo mucho más rápido y duradero. Es de recalcar, que 
si bien muchos partían del nivel 1 (las respuestas fueron clasificadas en 3 niveles) en la evaluación inicial de sus conocimientos evaluados a través del cuestionario pre-post; esté desapareció en la evaluación final. Con respecto al nivel 3 , de la misma manera pero a la inversa, este no se presentaba en los inicios de las respuestas, y finalmente fue el más presente en todas las cuestiones evaluadas. Estos aprendizajes se han puesto de manifiesto no obstante, en los resultados obtenidos en la evaluación de la asignatura, con muy buenos resultados. El clima va siendo más distendido cuando entienden, que sus aportaciones no son valoradas o cuestionadas, sino reforzadas o incitadas al replanteamiento.

Con respecto a las actividades estas han podido desarrollarse en su totalidad, ya que en este caso han estado bastante ajustadas a los horarios y contenidos de la asignatura, tanto las desarrolladas en grupo mediano como grupo pequeño. Además como la mayoría de las actividades se han realizado en el aula, y las que tenían que realizar en cas eran muy explícitas; ha permitido que el ritmo de trabajo sea constante y bueno.

En relación con respecto a la actividad docente, el haber desarrollado un Ciclo de Mejora anterior, aunque con menos horas, si me ha permitido sentirme con mayor capacidad de control ante el "desconcierto" que provoca en el alumnado "mantenerse en ocasiones con la boca cerrada". En cuanto a haber llevado a cabo el Diario, que es una nueva práctica que he implantado con respecto al ciclo anterior para seguir la evolución de los alumnos, ha sido muy interesante, ya que permite hacerse conscientes de cuestiones que hasta ahora no me había planteado, como realizar ciertas preguntas que aseguren que el alumnado ha asimilado los conceptos trabajados. Además, permite hacer un registro de las cosas que se han podido o no hacer con respecto a lo planteado en el inicio, así como de sesión en sesión, y poder ajustar sobre todo los tiempos a las temáticas. 


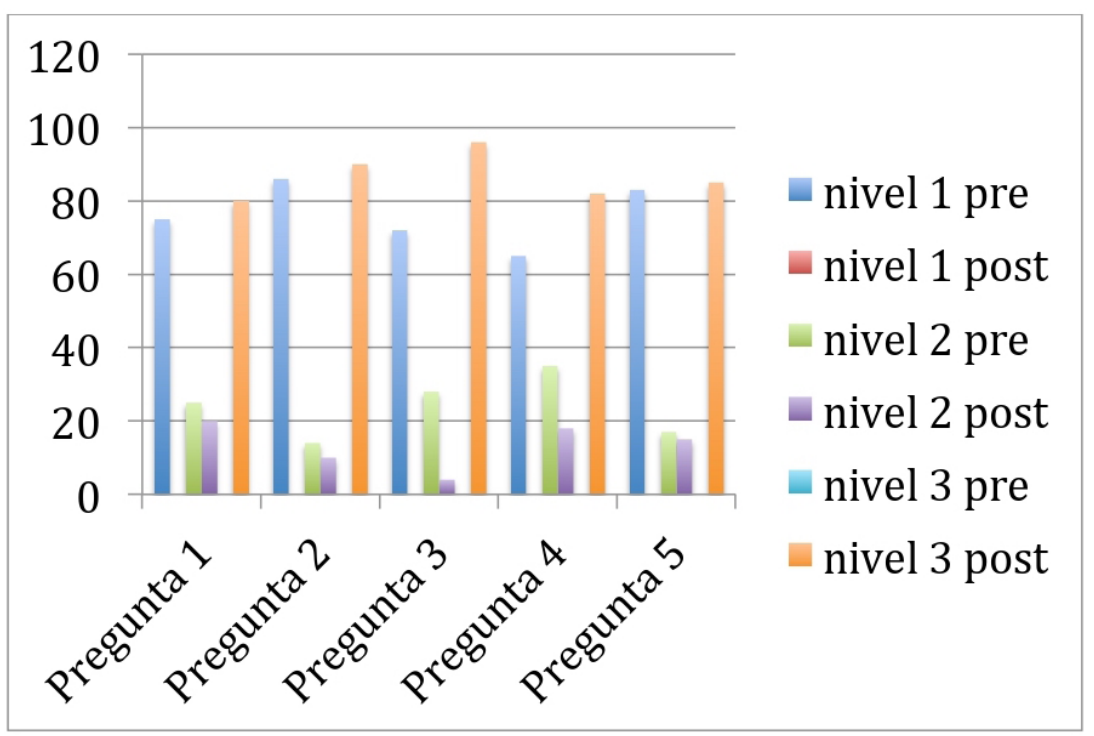

Figura 2. Escalera de aprendizaje. Los datos reflejan los porcentajes de respuestas recogidos en la Tabla 3

Tabla 3. Cuadro con datos en porcentajes de la evaluación pre y post del ciclo de mejora en los alumnos.

\begin{tabular}{|l|l|l|l|l|l|l|}
\hline PREGUNTA & $\begin{array}{l}\text { NIVEL 1 } \\
\text { PRE }\end{array}$ & $\begin{array}{l}\text { NIVEL 1 } \\
\text { POST }\end{array}$ & $\begin{array}{l}\text { NIVEL 2 } \\
\text { PRE }\end{array}$ & $\begin{array}{l}\text { NIVEL 2 } \\
\text { POST }\end{array}$ & $\begin{array}{l}\text { NIVEL 3 } \\
\text { PRE }\end{array}$ & $\begin{array}{l}\text { NIVEL 3 } \\
\text { POST }\end{array}$ \\
\hline $\begin{array}{l}\text { P.1 ¿Qué es } \\
\text { para ti la } \\
\text { memoria? } \\
\text { ¿Hay } \\
\text { diferentes } \\
\text { tipos? }\end{array}$ & 75 & 0 & 25 & 20 & 0 & 80 \\
\hline $\begin{array}{l}\text { P.2. ¿Qué } \\
\text { trastornos } \\
\text { de memoria } \\
\text { conoces? } \\
\text { Enuméralos } \\
\text { así como } \\
\text { intenta } \\
\text { describir sus } \\
\text { caracteristicas }\end{array}$ & 86 & 0 & 14 & 10 & 0 & 90 \\
\hline
\end{tabular}

Jornadas de Formación e Innovación Docente del Profesorado | № 2 (2019) Esta obra se distribuye con la licencia Creative Commons Reconocimiento-NoComercial-SinObraDerivada 


\begin{tabular}{|c|c|c|c|c|c|c|}
\hline $\begin{array}{l}\text { P.3 ¿Por qué } \\
\text { crees que hay } \\
\text { pérdida de } \\
\text { memoria en } \\
\text { las personas } \\
\text { mayores? ¿Y } \\
\text { en personas } \\
\text { de mediana } \\
\text { edad? ¿Y en } \\
\text { personas } \\
\text { que sufren } \\
\text { algún tipo } \\
\text { de "trauma" } \\
\text { (psicológico, } \\
\text { vascular, } \\
\text { accidental, } \\
\text { alcholismo...)? }\end{array}$ & 72 & 0 & 28 & 4 & 0 & 96 \\
\hline $\begin{array}{l}\text { P.4 ¿Cómo } \\
\text { sabrías si una } \\
\text { persona tiene } \\
\text { Alzheimer? }\end{array}$ & 65 & 0 & 35 & 18 & 0 & 82 \\
\hline $\begin{array}{l}\text { P.5 ¿Cómo } \\
\text { evaluarías e } \\
\text { intervendrías } \\
\text { a una } \\
\text { persona con } \\
\text { Alzheimer? }\end{array}$ & 83 & 0 & 17 & 15 & 0 & 85 \\
\hline
\end{tabular}

Jornadas de Formación e Innovación Docente del Profesorado I № 2 (2019) Esta obra se distribuye con la licencia Creative Commons 
ESPERANZA QUINTERO SÁNCHEZ

Tabla 4. Significado de los niveles de aprendizaje evaluados

\begin{tabular}{|c|c|c|c|c|c|c|}
\hline PREGUNTA & NIVEL 1 PRE & $\begin{array}{l}\text { NIVEL } 1 \\
\text { POST }\end{array}$ & NIVEL 2 PRE & NIVEL 2 POST & $\begin{array}{l}\text { NIVEL } \\
3 \text { PRE }\end{array}$ & NIVEL 3 POST \\
\hline $\begin{array}{l}\text { P.1 ¿Qué es para ti } \\
\text { la memoria? ¿Hay } \\
\text { diferentes tipos? }\end{array}$ & $\begin{array}{l}\text { Capacidad de } \\
\text { recordar. } \\
\text { Memoria a } \\
\text { corto y a largo } \\
\text { plazo }\end{array}$ & & $\begin{array}{l}\text { Capacidad de } \\
\text { aprender y } \\
\text { recordar. }\end{array}$ & $\begin{array}{l}\text { Memoria episódica, } \\
\text { semántica y } \\
\text { procedimental y } \\
\text { memoria a corto } \\
\text { plazo }\end{array}$ & & $\begin{array}{l}\text { Capacidad de aprender, almacenar y } \\
\text { recuperar información. } \\
\text { Memoria a largo plazo: m. Episódica, } \\
\text { semántica y procedimental, y memoria } \\
\text { de trabajo }\end{array}$ \\
\hline $\begin{array}{l}\text { P.2. ¿Qué trastornos } \\
\text { de memoria conoces? } \\
\text { Enuméralos así como } \\
\text { intenta describir sus } \\
\text { características }\end{array}$ & $\begin{array}{l}\text { Alzheimer, } \\
\text { pierden los } \\
\text { recuerdos }\end{array}$ & & $\begin{array}{l}\text { Alzheimer y } \\
\text { demencias; } \\
\text { pérdida de } \\
\text { memoria }\end{array}$ & $\begin{array}{l}\text { Alzheimer y } \\
\text { otras demencias } \\
\text { que afectan a } \\
\text { los diferentes } \\
\text { sistemas de } \\
\text { memoria }\end{array}$ & & $\begin{array}{l}\text { Alzheimer que van perdiendo todos los } \\
\text { sistemas de memoria den diferentes } \\
\text { fases, amnesia anterógrada y retrógrada, } \\
\text { que afecta a la memoria a corto y a la } \\
\text { larga respectivamente cada una con sus } \\
\text { características; y la demencia semántica, } \\
\text { que pierden los conceptos. }\end{array}$ \\
\hline $\begin{array}{l}\text { P.3 ¿Por qué crees } \\
\text { que hay pérdida } \\
\text { de memoria en las } \\
\text { personas mayores? } \\
\text { ¿Y en personas de } \\
\text { mediana edad? ¿Y } \\
\text { en personas que } \\
\text { sufren algún tipo de } \\
\text { "trauma” (psicológico, } \\
\text { vascular, accidental, } \\
\text { alcholismo...)? }\end{array}$ & Por la edad & & $\begin{array}{l}\text { Por la pérdida } \\
\text { de capacidades } \\
\text { psicológicas }\end{array}$ & $\begin{array}{l}\text { Deterioro de } \\
\text { capacidades fisicas } \\
\text { y psicológicas }\end{array}$ & & $\begin{array}{l}\text { Por la pérdida de capacidades cognitivas, } \\
\text { como atención, aprendizaje y memoria }\end{array}$ \\
\hline
\end{tabular}

Jornadas de Formación e Innovación Docente del Profesorado | № 2 (2019)

(c) (7) $\odot$ Esta obra se distribuye con la licencia Creative Commons

Internacional (CC BY-NC-ND 4.0.) 
ESPERANZA QUINTERO SÁNCHEZ

\begin{tabular}{|l|l|l|l|l|l|l|}
\hline $\begin{array}{l}\text { P.4 ¿Cómo sabrías si } \\
\text { una persona tiene } \\
\text { Alzheimer? }\end{array}$ & $\begin{array}{l}\text { Hablando } \\
\text { con ella, y } \\
\text { diciéndole } \\
\text { que me repita } \\
\text { lo que le voy } \\
\text { diciendo }\end{array}$ & & $\begin{array}{l}\text { En entrevista } \\
\text { semiestructurada }\end{array}$ & $\begin{array}{l}\text { Con entrevista y } \\
\text { evaluación }\end{array}$ & $\begin{array}{l}\text { Le pasaría pruebas para evaluar los } \\
\text { diferentes sistemas de memoria, como } \\
\text { recuerdo con claves para episódica, } \\
\text { que nombrase objetos para semántica, } \\
\text { completar raíces para procedimental, y } \\
\text { tareas concurrentes para memoria de } \\
\text { trabajo }\end{array}$ \\
\hline $\begin{array}{l}\text { P.5 ¿Cómo evaluarías } \\
\text { e intervendrías a } \\
\text { una persona con } \\
\text { Alzheimer? }\end{array}$ & $\begin{array}{l}\text { Hablando y } \\
\text { con preguntas. } \\
\text { No sé cómo } \\
\text { intervendría }\end{array}$ & $\begin{array}{l}\text { Con algunos } \\
\text { test, aunque no } \\
\text { conozco cuáles } \\
\text { hay }\end{array}$ & $\begin{array}{l}\text { Con las diferentes } \\
\text { tareas de } \\
\text { evaluación que } \\
\text { hemos visto }\end{array}$ & $\begin{array}{l}\text { Con las tareas que he mencionado en } \\
\text { la pregunta anterior, evaluando cada } \\
\text { sistema por separado para ver en qué } \\
\text { grado se encuentra; y así hacer un plan } \\
\text { de intervención adecuado }\end{array}$ \\
\hline
\end{tabular}

\section{Evaluación del CIMA}

Una visión general del CIMA, nos permite obtener información muy valiosa para seguir trabajando en futuras implantaciones de Ciclos, tanto ampliando en la asignatura que hemos tratado, como a implantar en otras asignaturas. Podría decir que haber desarrollado un Ciclo de Mejora anterior, aunque con menor alcance, facilita mucho la puesta en marcha de este nuevo Ciclo. Como puntos favorables del mismo, pueden decirse que los objetivos de partida con respecto al desarrollo de la asignatura y adquisición de conocimientos han sido conseguidos por el alumnado. Si bien, sería necesario extrapolar este tipo de enseñanza a las clases de Grupo Grande, dónde hay mucha más carga teórica, la cual se ve que no ha sido del todo aprovechada por los resultados obtenidos en la evaluación tradicional en examen de la asignatura. 
Sería interesante también, establecer actividades extras, para aquellos alumnos y alumnas que lo necesiten o demanden, y puedan realizar fuera del aula.

Además, plantear que al igual que en la realización de los informes experimentales se les explica y ofrece un documento con los puntos ha desarrollar y el contenido de los mismos (algo que ya conocen pues se sujeta a los criterios de publicación de artículos de investigación); hacer algo parecido con los casos prácticos, de modo que ellos tengan un guión o pautas. Si bien, esto es explicado y recordado en cada clase, podría facilitárseles o mejor aún, dedicar una sesión a construirlo en conjunto toda la clase.

Por otro lado, también sería interesante hacer partícipe al alumnado promoviendo que sean ellos los que propongan los casos tratados en los Grupos Pequeños; así siendo inventados o creados, como aportados ya hechos en distintos formatos (escritos, vídeos..). Así como, trabajar más con casos y formatos vistos en exámenes anteriores.

\section{Conclusiones}

Tras haber aplicado el Ciclo y evaluado los resultados obtenidos por los alumnos y alumnos así sea por el cuestionario de evaluación inicial-final de los contenidos planteados, como por el formato de evaluación recogido en la asignatura; cabe destacar que parece ser que ha sido de provecho para el alumnado la implantación del mismo. Si bien como docente también me ha permitido avanzar y abrir expectativas, reformular modelos aprendidos en el pasado.

También reafirmar que el modelo de aprendizaje elegido para llevar a cabo este Ciclo ha sido bien elegido, y que podría ser ampliado y aplicado a toda la asignatura al completo, durante todas las horas de docencia que comprende (86 horas). 
Por último recalcar, que no sólo se ha trabajado y desarrollado los contenidos conceptuales y procedimentales, sino que también se han podido ver reflejado los contenidos de tipo actitudinal, que serán los que en un futuro diferencie los "buenos" de los "malos" profesionales. 


\section{Referencias bibliográficas}

Bain, K. (2004). Traducción Barberá, O. (2008). Lo que hacen los mejores profesores universitarios. Universidad de Valencia. España: PUV.

Finkel, D. Traducción Barberá, O. (2008). Dar clases con la boca cerrada. Valencia. España: Universidad de Valencia. Libro de resúmenes del 1 Congreso Internacional de In- novación y Tendencias Educativas. INNTED (2017). Innovación y Tendencias Educativas. Sevilla. España: Universidad de Sevilla.

Porlán, R. (2017). Enseñanza Universitaria. Como mejorarla. Universidad de Sevilla. España: Ediciones Morata

Jornadas de Formación e Innovación Docente del Profesorado | № 2 (2019) Esta obra se distribuye con la licencia Creative Commons 